\section{Science Mimics Color Diffraction of Butterfly Wings}

The wings of the morpho butterfly have a multilayered structure that produces a metallic blue iridescent color through an interference effect. As reported in the December 1, 2003, issue of Optics Letters, researchers at the Applied Research Center of Old Dominion University, in collaboration with researchers at Alcoa Corporation, developed a method to artificially replicate these structures by specially designing grating structures fabricated by electron-beam lithography (EBL).

Normal ruled grating techniques are ineffective since they do not produce single colors that can be viewed over wide angles. Another method consists of removing a grating layer from a substrate and crushing it to form small flakes, but, as the researchers said, this technique is impractical because of the difficulty in controlling the exact size of the flakes; the technique, therefore, shows shifts in color with changing incident light and viewing angle. The structure fabricated by the director of the Applied Research Center Mool Gupta and co-workers contains many small hexagonal gratings arranged randomly in any of the six possible orientations. Each small grating diffracts the incident white light; the diffracted light beams, then, interfere with each other, producing blue light. The multigrating structures were manufactured by EBL in an electron-beam resist (PMMA 496) coated on a silicon substrate. The researchers calculated the optimum values of duty cycle and groove depth as well as the grating period to achieve the blue color. The grating period was $440 \mathrm{~nm}$ and the groove depth was $125 \mathrm{~nm}$. The grating line width, measured by atomic force microscopy, was $220 \mathrm{~nm}$.

An argon-ion laser beam directed normal to the structure produced a complex diffraction pattern on a screen placed parallel to the surface. The pattern can be used to analyze the diffraction mechanism and to calculate the distance between fringes. The researchers said their results will help stimulate theoretical studies of observed complex diffraction patterns.

Through their technique, the researchers produced blue color by diffraction of white light with a method inspired by the morpho butterfly's wings. As opposed to other fabrication methods reported so far, this

\title{
Chinese Terracotta Army Restored through Electron-Beam Polymerization
}

The polychrome terracotta army found in the tomb of the first Chinese Emperor Qin Shihuangdi, who died in 210 BC, presents a restoration and conservation problem to scientists. After 2200 years in the damp earth, the painted, lifelike figures lose their color soon after excavation, and established methods of stabilizing the colors have failed. Heinz Langhals, Daniela Bathelt, and Ingo Rogner from the University of Munich have devised a method involving electron-beam curing in order to restore the colors.

The first terracotta fragments were found in Lintong in 1974, and over 1500 of the life-sized warriors have been excavated. "The lacquer base coat has changed while in storage," said Langhals. "The foundation cracks, comes loose, rolls together, and falls off as soon as the relative humidity gets below $84 \%$." The layer of color on top of it, then, is also lost.

Langhals, who was confronted with the problem in 2002 during a research visit at the Bingmayong Museum in China, said that typical preservation materials cannot work their way through the very fine pore structure of the water-saturated base coat. As described in the researchers' article in the December 2003 issue of Angewandte Chemie International Edition, the polychrome consists of a base coat of qi-lacquer-most likely with additives such as rice starch-and a pigment layer containing, for example, cinnabar or malachite.

The researchers treated the excavated fragments with hydroxyethyl methacrylate (HEMA), a common monomer used in the production of plastics, in the presence of the cross-linker polyethylene glycol dimethacryl ester (PEG-DMA 330). Since both are water soluble, they can be used directly on the damp terracotta. Irradiation with electron beams went straight through the lacquer layer, but stopped at the terracotta, hardening the HEMA all the way through the laquer layers.

"The cross-linking thus begins with high intensity at the adherence-critical terracotta-lacquer interface and continues toward the outer surface," said Langhals. "The reaction is stopped by oxygen from the surrounding air at the surface, preventing it from becoming shiny, which would greatly disturb the natural look of the clay warriors."

multigrating structure appears blue over broad viewing angles, similar to those observed in butterfly wings. Furthermore, said the research team, by choosing grating size, orientation, groove density, and depth, both color and efficiency of the diffraction light can be controlled.

\section{MARIA M. CORTALEZZI}

\section{Green-Emitting Lanthanoid Nanocrystals with Core-and-Shell Structure Demonstrate High- Intensity Luminescence}

Nanoscale luminescent crystals are in demand because they can be used as active components in monitors, light-emitting diodes, and optical intensifiers, and also as markers for the investigation of biomolecules. One of the well-known techniques of increasing quantum efficiency used in semiconducting nanoparticles is growing a crystalline shell of a suitable large-bandgap material around nanocrystals. By applying this technique to lanthanide(III) phosphate materials, M. Haase of the University of Hamburg, A. Lobo of HASYLAB/DESY in Hamburg, and their colleagues developed green-emitting $\mathrm{CePO}_{4}: \mathrm{Tb} / \mathrm{LaPO}_{4}$ coreshell nanoparticles.

As reported in the November 2003 issue of Angewandte Chemie International Edition, the research team prepared $\mathrm{CePO}_{4}: \mathrm{Tb}$-doped nanoparticles with an average diameter of $4 \mathrm{~nm}$ by reaction of RE chlorides with phosphoric acid in nonaqueous media. The nanocrystals provide a rigid crystal environment for the dopant ions, which would ideally lead to a high quantum yield; however, as a result of energy-transfer processes to the surface, the quantum yield decreases. To suppress the energy-loss processes at the surface of the nanoparticles, the researchers used a 2-nm-thick shell of undoped $\mathrm{LaPO}_{4}$, which has a similar lattice constant to cerium phosphate, to avoid the formation of defects at the core-shell interface.

"While the excitation energy can be transferred from one cerium atom to another, this energy transport doesn't work between cerium and lanthanum," said Haase. "The lanthanum phosphate layer thus functions as a barrier to energy transport to the surface of the nanocrystals."

According to fluorescence analysis, the research team obtained a total quantum efficiency, which is a measurement of how much of the incoming UV light is converted into luminescence, of $53 \%$ for dilute colloidal solutions of $\mathrm{CePO}_{4}: \mathrm{Tb}$ particles and a quantum efficiency of $\sim 80 \%$ for $\mathrm{CePO}_{4}: \mathrm{Tb} / \mathrm{LaPO}_{4}$ core-shell nanoparticles. By $\mathrm{x}$-ray photoelectron spectroscopy, the researchers verified the 